\title{
Dexmedetomidine alleviates pulmonary ischemia-reperfusion injury through modulating the miR-21-5p/Nr4a1 signaling pathway
}

\author{
Wei Dong1', Hongxia Yang'2, Minghua Cheng ${ }^{1}$, Xin Zhang ${ }^{3}$, Jingjing Yin ${ }^{4}$, Zhaodong Zeng1ه \\ and Guang Huang ${ }^{5}$
}

1Department of Anesthesiology, The First Affiliated Hospital of Shantou University Medical College, Shantou City, Guangdong Province, 515041, China; ${ }^{2}$ Department of Sector 2 of Hepatic and Gallbladder Surgery, The First Affiliated Hospital of Shantou University Medical College, Shantou City, Guangdong Province, 515041, China; ${ }^{3}$ Department of Molecular Biology Laboratory, The First Affiliated Hospital of Shantou University Medical College, Shantou City, Guangdong Province, 515041, China; ${ }^{4}$ Department of Radiology, The First Affiliated Hospital of Shantou University Medical College, Shantou City, Guangdong Province, 515041, China; ${ }^{5}$ Department of paediatrics, The First Affiliated Hospital of Shantou University Medical College, Shantou City, Guangdong Province, 515041, China

This study aims to investigate the protection of dexmedetomidine (Dex) against pulmonary ischemia-reperfusion injury (PIRI) in the mouse model and reveal the mechanism in hypoxia reoxygenation (H/R)-induced mouse pulmonary vascular endothelial cells (MPVECs). The lung wet-to-dry weight ratio, histopathological features, and malondialdehyde (MDA) concentrations were measured. The H/R-induced MPVECs were exposed to Dex, and the cell viability, cell apoptosis and protein expressions were assessed by the Cell Counting Kit- 8 (CCK8) assay, flow cytometry and western blot, respectively. In addition, the regulatory relationship between miR-21-5p and orphan nuclear receptor 4A1 (Nr4a1) was revealed by several assays, including the dual-luciferase reporter assay, real-time quantitative polymerase chain reaction (RT-qPCR) and western blot. We found that the Dex treatment significantly alleviated pulmonary injury and decreased the level of MDA and wet/dry weight ratio in PIRI mice. Dex treatment also increased cell viability, reduced apoptotic ratio and downregulated expression levels of Cleaved Caspase- 3 and Cleaved Caspase-9 in $H / R$ induced MPVECs. Furthermore, the expression of miR-21-5p was upregulated, while Nr4a1 was downregulated by Dex in a concentration-dependent manner in $\mathrm{H} / \mathrm{R}$ induced MPVECs. Moreover, Nr4a1 was verified as a target of miR-497-5p. Overexpression of Nr4a1 could reverse the protective effects of Dex on alleviating H/R-induced injury in MPVECs. Taken together, Dex treatment attenuated ischemia-reperfusion induced pulmonary injury through modulating the miR-21-5p/Nr4a1 signaling pathway.

Key words: Pulmonary ischemia-reperfusion injury, dexmedetomidine, mouse pulmonary vascular endothelial cells, MiR-21-5p, the orphan nuclear receptor $4 \mathrm{~A} 1$

Received: 27 May, 2020; revised: 27 July, 2020; accepted: 31 August, 2020; available on-line: 17 December, 2020

『e-mail: ZhaodongZengsjk@163.com

Acknowledgements of Financial Support: This work was supported by Shantou science and technology plan medical and health category project (Grant No. 181126204017535)

Abbreviations: CCK8, Cell Counting Kit-8; Dex, dexmedetomidine FBS, fetal bovine serum; H\&E, Hematoxylin and eosin; H/R,hypoxia reoxygenation; MDA, malondialdehyde; miRNAs, MicroRNAs; miR21-5p, microRNA-21-5P; MPVECs, mouse pulmonary vascular endothelial cells; MUT, mutation type; NC, negative control; Nr4a1, orphan nuclear receptor 4A1; PIRI, Pulmonary ischemia-reperfusion injury; RT-qPCR, real-time quantitative polymerase chain reaction; UTR, untranslation region; WT, wild type; W/D, wet/dry

\section{INTRODUCTION}

Pulmonary ischemia-reperfusion injury (PIRI) is a kind of aseptic acute pulmonary injury which is presented in many pathological conditions and medical situations, including pulmonary embolism (Clarke et al., 2002), hemorrhagic shock (Oda et al., 2002), trauma (Wang et al., 2018), pulmonary transplantation (Sugimoto et al., 2019) and open-heart surgery (Amour et al., 2019). This event is a common surgical complication that can lead to pulmonary dysfunction and reduce the survival rate of patients (den Hengst et al., 2010). Studies have shown that the pathogenesis of PIRI is complex, involving many pathophysiological processes and pathways, such as apoptosis (Wagner et al., 2006), autophagy (Zhang \& Zhang, 2017), lymphocyte and macrophage infiltration (van der Kaaij et al., 2009) in oxidative stress injury (Yu et al., 2019), calcium overload (Li et al., 2015) and inflammatory injury (Krishnadasan et al., 2002). Therefore, many pharmacological agents involved in these pathophysiological processes and pathways were studied to alleviate PIRI, for instance, dexmedetomidine (Dex) (Liang et al., 2019), pirfenidone (Saito et al., 2019) and dexamethasone (Zhou et al., 2012).

In particular, Dex is a highly potent and selective $\alpha 2$-adrenergic agonist which has multiple pharmacological functions, such as anxiolysis, sedation, antioxidant, anti-inflammatory and anti-apoptosis (Vora et al., 2015; Wang et al., 2015). Recent studies have suggested that Dex could alleviate ischemia-reperfusion injury of different organs (Yuan et al., 2017; Ren et al., 2018; Liu et al., 2019). Wang, et al., found that Dex may inhibit neuronal apoptosis via inhibition of hypoxia-inducible factor-1alpha to alleviate cerebral ischemia-reperfusion injury in rats (Wang et al., 2018). Ma, et al., indicated that Dex displayed renoprotection in renal ischemia-reperfusion injury, and acted as an anti-inflammatory effector to the parasympathetic nervous system activation (Ma et al., 2018). Though the previous studied have reported that Dex could reduce PIRI by its anti-apoptotic effects (Zhang et al., 2016; Kucukebe et al., 2017; Xue et al., 2019), the exact mechanisms underlying the effects of Dex on reducing PIRI remain to be further studied.

MicroRNAs (miRNAs) are non-coding RNAs of 22-25 nucleotides in length and play a negative role in post-transcriptional gene expression. In previous studies, it was shown that many microRNAs play an important 
role in ischemia-reperfusion injury (Zhao et al., 2015; Huang et al., 2019; Zhang et al., 2019). For example, miR-496 could reduce cerebral ischemia-reperfusion injury by inhibiting the BCL2L14 expression (Yao et al., 2019). And miR-219a-5p, an age-related miRNA, was reported to attenuate hepatic ischemia-reperfusion injury by inhibition of TP53BP2 in mice (Xiao et al., 2019). Importantly, miR-21-5p, acting as an anti-apoptotic agent, has been reported to mediate mesenchymal stromal cellsecreted exosomes to relieve ischemia-reperfusion injury in mice (Li et al., 2019). However, the role of miR-21-5p in PIRI is still unclear.

In this study, the PIRI mouse model was established and mouse pulmonary vascular endothelial cells (MPVECs) were treated with hypoxia reoxygenation $(\mathrm{H} / \mathrm{R})$ to investigate the effect of Dex on pulmonary reperfusion injury. Also, TargetScan was used to predict biological targets of miR-21-5p, and $\mathrm{Nr} 4 \mathrm{a} 1$ was identified. In addition, further rescue experiments proved that overexpression of $\mathrm{Nr} 4 \mathrm{a} 1$, as a target of miR21-5p, could block the effect of Dex on H/R-treated MPVECs. Thus, the function and mechanism of Dex in PIRI was investigated in this study.

\section{MATERIALS AND METHODS}

Drugs and reagents. Dexmedetomidine hydrochloride (Dex) was purchased from Sigma Aldrich (St Louis, MO, USA). Dex was dissolved in normal saline at a 20 $\mathrm{mg} / \mathrm{mL}$ concentration as a stock solution. The Nr4a1WT or Nr4a1-MUT reporter plasmids were constructed by cloning the wild type or mutated miR-21-5p binding sequence into the pGL3 reporter plasmids (GeneScript, Nanjing, China). The Nr4a1 cDNA fragment was cloned into pcDNA3.1 (Life Technologies, Darmstadt, Germany) to construct the $\mathrm{Nr} 4 \mathrm{a} 1$ overexpression vector. All of the constructed plasmids were verified by sequence analysis (not shown). In addition, the Nr4a1 overexpression vector was verified by qRT-PCR. The mimics of miR-21-5p, miR-21-5p inhibitor and negative control RNA were obtained from GenePharma (Shanghai, China). Other reagents involved in the study were acquired from a reputed vendor.

Cell lines. Mouse pulmonary vascular endothelial cells (MPVECs) (cat. no. BNCC340787) were purchased from the $\mathrm{BeNa}$ Culture Collection (BNCC). Human embryonic kidney cancer cells (HEK293) (cat. no. GNHu43) were purchased from the Cancer Institute of Chinese Academy of Medical Science. MPVECs and HEK293 cells were cultured at $37^{\circ} \mathrm{C}$ in Dulbecco's Modified Eagle's Medium (Hyclone, Logan, UT, USA) supplemented with 10\% Fetal Bovine Serum (FBS) and $100 \mathrm{U} / \mathrm{ml}$ penicillin-streptomycin and were cultivated in a humidified atmosphere containing $5 \% \mathrm{CO}_{2}$ in the air.

Animals. This study was conducted at the First Affiliated Hospital of Shantou University Medical College with the consent from the Experimental Animals Ethics Committee of the First Affiliated Hospital of Shantou University Medical College. The permission number for animal study is SUMC2019-310. All procedures were performed according to the Guides for the Care and Use of Laboratory Animals.

Pulmonary ischemia-reperfusion injury model. Healthy female clean grade C57BL/6J mice (6 to 8 -week-old) with body weight of $22-25 \mathrm{~g}$ were involved to establish the PIRI mice model as previously described (Cao et al., 2015). Briefly, anesthesia was performed with air containing $1 \%$ isoflurane, through tracheostomy, and we then intubated a mouse with intravenous puncture needle and mechanically ventilated the mouse with an oxygen rich atmosphere by a small animal respirator. The mouse was placed on the right side, and the chest was cut through the 5 th intercostal space through the left thoracic incision. After exposing the left pulmonary, we blocked the left pulmonary hilum with a non-invasive microvascular clip, resulting in pulmonary ischemia for 30 minutes. The microvascular clip was removed, and pulmonary blood perfusion was restored for 2 hours.

In this study, 24 mice were randomly divided into the Sham group, ischemia reperfusion (I/R) group, Sham + Dex group and ischemia reperfusion $(\mathrm{I} / \mathrm{R})+$ Dex group ( $\mathrm{n}=6$ in each group). Mechanical ventilation was performed without ischemia reperfusion in the Sham group. In the treatment group (Sham+Dex group and I/ $\mathrm{R}+$ Dex group), $20 \mu \mathrm{g} / \mathrm{kg}$ of Dex $(100 \mu \mathrm{g} / \mathrm{mL})$ was injected through the caudal vein.

Pulmonary tissue wet/dry (W/D) weight ratio. The W/D ratio was determined as previously described (Jiang et al., 2014). After pulmonary blood flow reperfusion for 2 hours, the left pulmonary was taken and weighed immediately to obtain the wet weight of pulmonary tissue. Next, we dried the pulmonary in an oven at $60^{\circ} \mathrm{C}$ for 48 hours to measure the dry weight. The W/D ratio was used to assess the pulmonary water content.

Hematoxylin and eosin $(\mathbf{H} \& \mathbf{E})$ staining. The pulmonary tissues were dissected from the mice in different groups as described (Naderali et al., 2018). Briefly, the pulmonary tissues were fixed by dipping in $4 \%$ paraformaldehyde for 48 hours, then the tissues were embedded in paraffin and cut into 3-4 $\mu \mathrm{m}$ thick slices. Finally, hematoxylin and eosin staining (H\&E) was performed to observe the pathological changes in pulmonary tissues under a microscope. Five fields of view were randomly selected under $200 \times$ magnifications and the number of alveolar cells containing more than two red blood cells or white blood cells was counted, and the alveolar damage index was calculated.

Measurements of MDA levels in pulmonary tissues. The pulmonary tissues were harvested and immediately homogenized on ice in 5 volumes of normal saline. Then, the supernatant was collected after homogenates' centrifugation at $1200 \times g$ for 10 minutes. The MDA levels were measured in the supernatant according to the manufacturer's manual of a lipid peroxidation (MDA) Assay Kit (Biovision, San Francisco, USA).

Hypoxia reoxygenation $(\mathbf{H} / \mathbf{R})$ model. The $H / R$ model was performed as previously described (Sauvant et al., 2009). Briefly, for hypoxia treatment, the MPVECs were cultured in an anaerobic chamber equilibrated with $5 \% \mathrm{CO}_{2}$ and $95 \% \mathrm{~N}_{2}$ for 4 hours, and then maintained in a constant low oxygen concentration $(<0.1 \%)$ chamber. After the hypoxia treatment, the MPVECs were moved back to a normal incubator with $5 \% \mathrm{CO}_{2}$ in the air. The treatment group was treated with different concentrations of Dex ( $1 \mu \mathrm{M}, 5 \mu \mathrm{M}, 10 \mu \mathrm{M}$ or $20 \mu \mathrm{M})$.

Cell transfection. HEK293 and MPVECs were seeded at a density of $5 \times 10^{5}$ cells/well in 6-well culture plates (NEST, CA, USA). When the cell fusion rate reached $70 \%$, the cells were transfected with miR-21$5 \mathrm{p}$ mimic $(40 \mathrm{nM})$, inhibitor $(55 \mathrm{nM})$ or $\mathrm{Nr} 4 \mathrm{a} 1$ expression vector $(1 \mu \mathrm{g})$ and their corresponding controls using Lipofectamine 3000 (Invitrogen, CA, USA). After 4 hours, we discarded the transfection reagent and washed the cells with DMEM, then added fresh DMEM medium contain 10\% FBS and different concentration of Dex $(1 \mu \mathrm{M}, 5 \mu \mathrm{M}, 10 \mu \mathrm{M}) .24$ hours after transfection, the cells were harvested for further analysis. 
Cell viability. To assay cell viability, the Cell Counting Kit-8 (CCK8) assay (Beyotime Biotechnology, Shanghai, China) was employed according to the manufacturer's manual. We seeded a total of $5 \times 10^{3}$ treated MPVECs in 96-well plates with $90 \mu \mathrm{L}$ DMEM medium containing $10 \%$ FBS. Then, $10 \mu \mathrm{L}$ of the CCK8 solution was added each well, and cells were incubated for 2 hours at $37^{\circ} \mathrm{C}$ at each indicated time point. Absorbance was then recorded at $450 \mathrm{~nm}$ using cytation 5 (BioTex VT, USA). Each experiment was repeated at least three times, and the mean of all measurements was calculated. The absorbance at each time point was used to plot the cell viability.

Apoptosis analysis. The Annexin V-FITC/PI apoptosis kit (eBioscience, UAS) was used to measure cell apoptosis according to the manufacturer's recommendations. Briefly, 24 hours after transfection, the MPVECs were harvested and washed with PBS at least two times. Then, the cells were resuspended in the binding cocktail containing Annexin V and PI, and kept in the dark for $15 \mathrm{~min}$ at room temperature. BD C6 flow cytometer (BD Biosciences, California, USA) was used to detect the stained cells. The results were analyzed by the Cell Quest software (BD Biosciences).

Luciferase reporter assay. MPVECs and 293T cells were co-transfected with $0.24 \mu \mathrm{g}$ of Nr4a1-WT or Nr4a1-MUT reporter plasmids, together with $40 \mathrm{nM}$ of miR-21-5p mimics or negative control oligoribonucleotides using Lipofectamine3000 (Invitrogen, USA) and following the manufacturer's instructions. In addition, $0.05 \mu \mathrm{g}$ of Renilla luciferase expression plasmids were transfected into cells as a reference control, and 36 hours after transfection the cells were harvested to detect the firefly and renilla luciferase activities by dual luciferase reporter assays (Promega, E1910, WI, USA). The relative luciferase activity was calculated as a ratio of firefly and renilla luciferase activities.

Quantitative real-time polymerase chain reaction (qRT-PCR). According to the manufacturer's instructions, total RNA from the pulmonary tissues and cultured cells were extracted by the TRIzol reagent (Invitrogen). Then, the RNAs were reverse transcribed into cDNA using the PrimeScript RT Reagent (TaKaRa, Japan). Finally, PCR was performed to detect the relative expression level of miRNA and mRNA. To normalize expression of miR-21-5p, U6 small nuclear RNA (snRNA) was used as an internal control, while GAPDH was used to normalize the expression of $\mathrm{Nr} 4 \mathrm{a} 1$, and the ${ }^{2}{ }^{\Delta \Delta} \mathrm{CT}$ equation was used for calculating relative expression.

Primers for miR-21-5p, U6, NR4A1 and GAPDH were as follows (Zhu et al., 2017; Ren et al., 2019):

RT primer for miR-21

GTCGTATCCAGTGCACGCTCCGAGGTATTCGCACTGGATACGACTCAACATC

5'-TAGCTTATCAGACTGATGTTGA-3'

(miR-21-5p, forward);

5'-TGCGTGTCGTGGAGT-3' (miR-21-5p, reverse);

5'-CTCGCTTCGGCAGCACA-3' (U6, forward);

5'-AACGCT'TCACGAATTTTGCGT-3' (U6, reverse).

5'-CAGCTTGCTTGTCGATGTC-3' (NR4A1, forward);

5'-GTGTCCATGAAGATCTTGTCAATG-3'

(NR4A1, reverse);

5'-CGAGCCACATCGCTCAGACA-3' (GAPDH, forward);

5'-GTGGTGAAGACGCCAGTGGA-3' (GAPDH, reverse).

Western blot analysis. The pulmonary tissues and cultured cell samples were harvested in a lysis buffer, the samples were incubated on ice for $30 \mathrm{~min}$ and the super- natant was collected by centrifugation at $1.5 \times 10^{4} \mathrm{~g}$ for 5 $\min$ at $4^{\circ} \mathrm{C}$. Then, we separated the total proteins $(40 \mu \mathrm{g})$ on $12 \%$ SDS-PAGE and transferred the proteins onto PVDF membranes (Millipore) via a MiniGenie blotting system (Bio-Rad). Next, we blocked the membranes with TBST containing 1\% skim milk powder for 1 hour and then incubated with rabbit monoclonal antibodies against murine Cleaved Caspase-9 (1:1000; cat. no. 20750S; Cell Signaling Technology), Cleaved Caspase-3 (1:1 000; cat. no. 9579S; Cell Signaling Technology), GAPDH (1:1 000; cat. no. 5174S; Cell Signaling Technology), and rabbit polyclonal antibody NR4A1 (1:2000; cat. no. PA5-27274; Invitrogen) at $4^{\circ} \mathrm{C}$ overnight. Next, the membranes were washed 3 times with TBST for 15 minutes, and then incubated with goat-anti-rabbit secondary antibodies (1:10000; cat. no. 14708S; Cell Signaling Technology) for 1 hour at room temperature. Finally, the protein bands were visualized on the membranes with an enhanced chemiluminescence system (ECL) and Image J (National Institutes of Health, Bethesda, MD) was used to quantify the protein bands.

Statistical analysis. GraphPad Prism (Version 6.01 for Windows) statistical software was used to perform statistical analysis. Student's $t$-test was used to compare the significant differences between groups. Repeated measures ANOVA was used for intragroup analysis. Statistical significance was set at $p<0.01$.

\section{RESULTS}

\section{Dex reduced the I/ R-induced pulmonary injury}

To explore the function of Dex in I/R-induced pulmonary injury, mice were injected with Dex during I/R operation through the caudal vein. The $\mathrm{W} / \mathrm{D}$ ratio of the pulmonary tissues was significantly higher in the I/R group when compared to the Sham group. The W/D ratio of the pulmonary tissues was significantly lower in the Sham+Dex group than in the $\mathrm{I} / \mathrm{R}+\mathrm{Dex}$ group. Importantly, as compared with the I/R group, the W/D ratio of the pulmonary tissues was decreased in the I/ $\mathrm{R}+$ Dex group $(p<0.01)$ (Fig. 1A). The H\&E staining analysis was performed to determine the histopathological changes, and the result revealed that the I/R operation caused more pulmonary injury as compared with the Sham group. In the presence of Dex, disruption of the histoarchitecture was attenuated in the $I / R+D e x$ group when compared to the $\mathrm{I} / \mathrm{R}$ group $(p<0.01)$ (Fig. 1B). Measurement of MDA level in the pulmonary tissue could reflect the degradation of lipids caused by oxidative stress, which is associated with the changes in the structure and the permeability of pulmonary membrane. As shown in Fig. 1C, the MDA level in the I/R group was the highest, while Dex treatment $(\mathrm{I} / \mathrm{R}+\mathrm{Dex})$ significantly reduced the MDA level when compared to the I/R group $(p<0.01)$. These results demonstrated the protective role of Dex in the I/R- induced pulmonary injury.

\section{Dex reduced the H/R-induced apoptosis of MPVECs}

To further study the role of Dex in reducing the I/Rinduced pulmonary injury, H/R-treated MPVECs were administered with Dex. The CCK8 assay showed that the $H / R$ treatment significantly reduced cell viability of MPVECs when compared to the control group. However, as compared to that in the $\mathrm{H} / \mathrm{R}$ group, the Dex treatment $(\mathrm{H} / \mathrm{R}+\mathrm{Dex})$ showed significantly increased vi- 
A

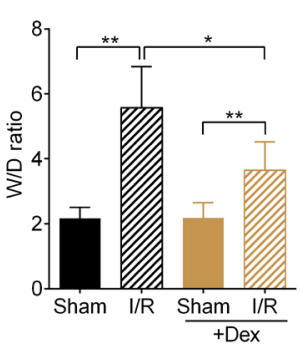

B



C

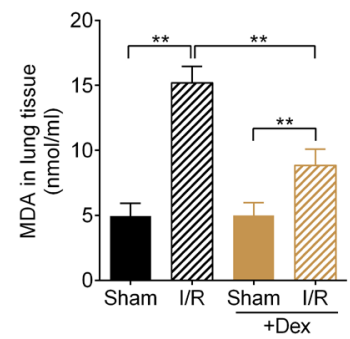

Figure 1. Effect of Dex on I/R-induced pulmonary injury.

(A) The pulmonary wet/dry ratio was assessed in different groups. (B) The histopathological changes in pulmonary tissues were determined by H\&E staining (magnification 200x). Scale bar was $100 \mu \mathrm{m}$. (C) Changes in pulmonary MDA levels (nmol/ml) in different groups. The results are representative of three independent experiments. The values represent mean $\pm S . D$. $\left(n=6\right.$ for each group). ${ }^{* *} p<0.01$, ${ }^{*} p<0.05$.

A



$\mathrm{B}$

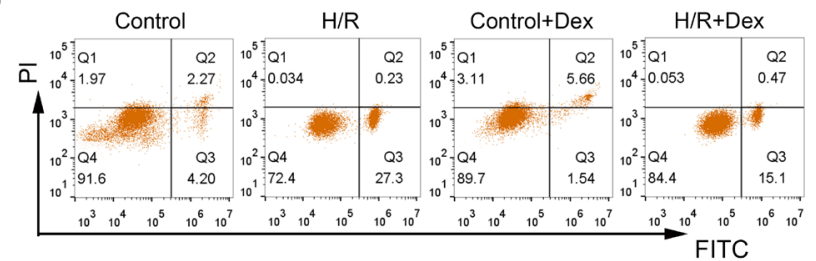

C


Figure 2. Effect of Dex on H/R-induced apoptosis of MPVECs

(A) CCK-8 assay was used to detect cell viability. (B) The cells were subjected to the FACS analysis to determine the cell apoptotic ratio. (C) The protein levels of cleaved caspase- 3 and cleaved caspase- 9 in pulmonary tissues were determined by western blot analysis with the corresponding antibodies. The results are representative of three independent experiments. The values represent mean \pm S.D. ( $n=3$ for each group). ${ }^{* *} p<0.01,{ }^{*} p<0.05$.

ability ( $p<0.01)$ (Fig. 2A). In addition, Annexin V-FITC/ PI double staining using flow cytometry was performed to detect cell apoptosis. As shown in Fig. 2B, when compared to the control group, the apoptotic ratio was greatly increased in the $\mathrm{H} / \mathrm{R}$ group $(p<0.01)$, while the Dex treatment $(\mathrm{H} / \mathrm{R}+\mathrm{Dex})$ reduced the apoptotic ratio $(p<0.01)$. Levels of cell apoptosis-related proteins were detected by western blot analysis. Consistently, the protein levels of Cleaved Caspase- 3 and Cleaved Caspase-9 in the $\mathrm{H} / \mathrm{R}$ group were greatly increased as compared to those in the control group $(p<0.01)$, and the Dex treatment $(\mathrm{H} / \mathrm{R}+\mathrm{Dex})$ decreased expression levels of Cleaved Caspase-3 and Cleaved Caspase-9 (Fig. 2C). These findings indicated that Dex could reduce $\mathrm{H} / \mathrm{R}$-induced apoptosis of MPVECs.

\section{Dex upregulated miR-21-5p level and decreased Nr4a1 expression in H/R-treated MPVCs}

Previous reports have described that miR-21-5p participated in the alleviation of I/R-induced injury in mouse $(\mathrm{Li}$ et al., 2019). TargetScan database was used and predicted that Nr4a1 was a putative target for miR-21-5p (Fig. 4A). qRT-PCR and western blot analysis were performed to detect the expression levels of miR-21-5p and Nr4a1 in
H/R- induced MPVECs at different times $(3 \mathrm{~h}, 6 \mathrm{~h}, 12$ $\mathrm{h}$ and $24 \mathrm{~h})$. The results showed that in the H/R-treated MPVECs, miR-21-5p was downregulated in a time-dependent manner (Fig. 3A). In contrast, Nr4a1 was upregulated in the H/R-treated MPVECs in a time-dependent manner (Fig. 3B). However, in the H/R-treated MPVECs, miR-21-5p was dose-dependently upregulated and $\mathrm{Nr} 4 \mathrm{a} 1$ was downregulated by the Dex treatement (all $p<0.01$ ) (Fig. 3C and Fig. 3D). Taken together, Dex could upregulate the expression of $\mathrm{miR}-21-5 \mathrm{p}$ and downregulate the expression of $\mathrm{Nr} 4 \mathrm{a} 1$ in $\mathrm{H} / \mathrm{R}$-treated MPVCs.

\section{Dex inhibited Nr4a1 expression via upregulation of miR-21-5p}

To verify the relationship between $\mathrm{Nr} 4 \mathrm{a} 1$ and miR21-5p, HEK293 cells were co-transfected with miR-21$5 \mathrm{p}$ mimic or miR-21-5p-inh and luciferase reporter plasmids containing Nr4a1-WT or Nr4a1-MUT (Fig. 4A). The data from qRT-PCR showed that miR-21-5p mimic increased the relative miR-21-5p level, while miR-21-5pinh decreased its level (Fig. 4B). The luciferase reporter assay showed that miR-21-5p mimics significantly decreased the luciferase activity of $\mathrm{Nr} 4 \mathrm{a} 1-\mathrm{WT}$ reporter when compared to the control group $(p<0.01)$. While 
A

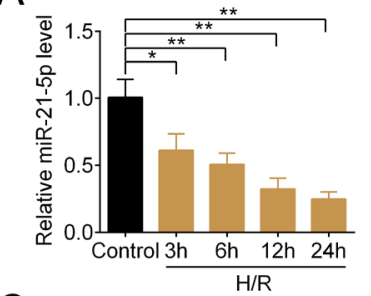

C

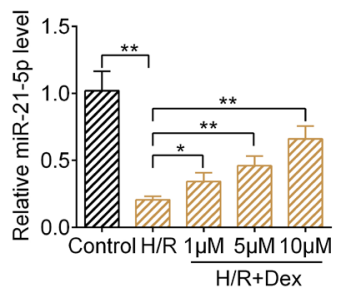

B
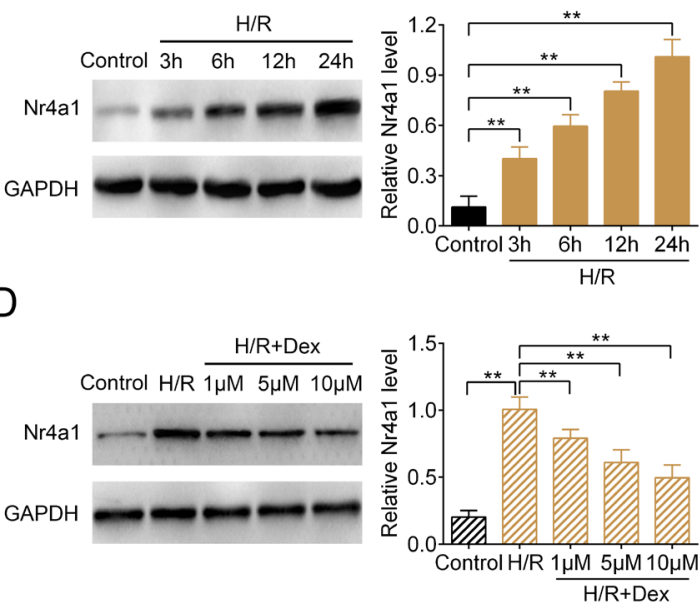

Figure 3. Effect of Dex on miR-21-5p and Nr4a1 expression in H/R induced MPVECs.

Relative expression level of miR-21-5p at different times ( $3 \mathrm{~h}, 6 \mathrm{~h}, 12 \mathrm{~h}, 24 \mathrm{~h})(\mathbf{A})$ or various Dex concentrations $(1 \mu \mathrm{M}, 5 \mu \mathrm{M}, 10 \mu \mathrm{M})(\mathbf{C})$ was detected by quantitative real-time reverse transcriptase polymerase chain reaction (qRT-PCR). Relative expression level of Nr4a1 at different times (3 h, 6 h, 12 h, 24 h) (B) or various Dex concentration groups $(1 \mu \mathrm{M}, 5 \mu \mathrm{M}, 10 \mu \mathrm{M})(\mathbf{D})$ was detected by western blot analysis with corresponding antibodies. The results are representative of three independent experiments. The values represent mean \pm S.D. ( $\mathrm{n}=3$ for each group). ${ }^{* *} p<0.01,{ }^{*} p<0.05$.

A $\begin{array}{cc}\text { Nr4a1-WT } & 5 \text {....ACCACUCUGUUGUAAAUAAGGUG...3' } \\ \text { miR-21-5p } & \text { 3'AGUUGUAGUCAGACUAUUCGAU 5' } \\ \text { Nr4a1-MUT } & 5^{\prime} \text {...ACCACUCUGUUGUAAUAUUCGAG...3' }\end{array}$

B

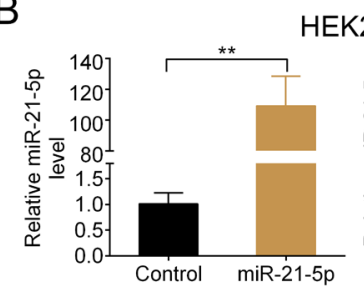

C

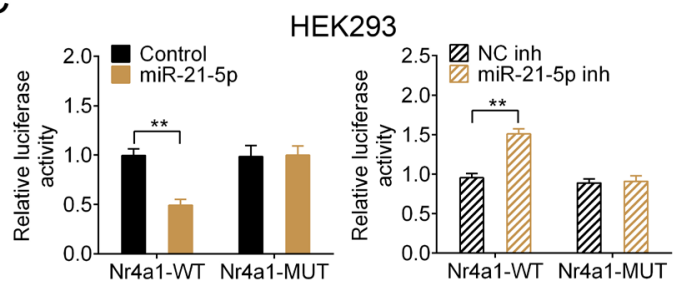

D

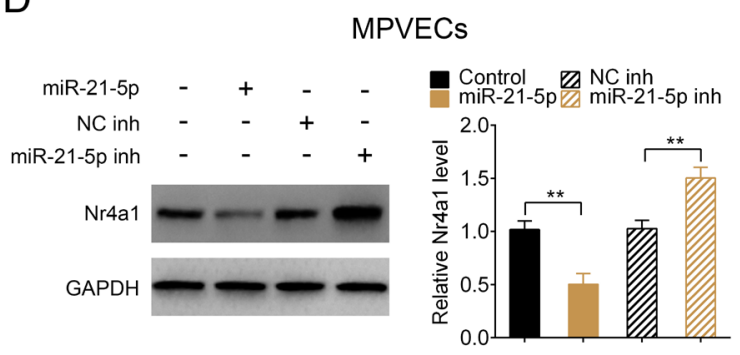

$\mathrm{E}$

\section{MPVECS}

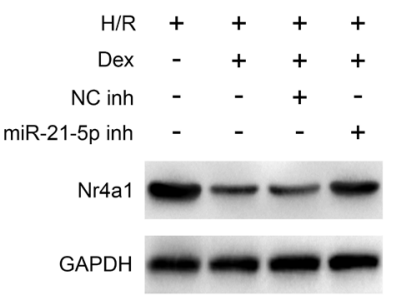

Figure 4. Involvement of miR-21-5p in the effect of DEX on H/R-treated MPVECs.

(A) The binding sequences between Nr4a1 and miR-21-5p. (B) Relative expression level of miR-520-3p in HEK293 cells after DEX (10 $\mu$ M) treatment for $24 \mathrm{~h}$ was detected by quantitative real-time reverse transcriptase polymerase chain reaction (qRT-PCR). (C) The relative luciferase activities in HEK293 cells that were co-transfected with wild-type (WT) 3'-untranslated region (3'-UTR) of Nr4a1 or mutant-type (MUT) 3'-UTR of Nr4a1 and miR-21-5p mimics/inhibitor. (D and E) Relative expression level of Nr4a1 in MPVECs was detected by western blot analysis with corresponding antibodies. The results are representative of three independent experiments. The values represent mean \pm S.D. ( $\mathrm{n}=3$ for each group). ${ }^{* *} p<0.01,{ }^{*} p<0.05$.

miR-21-5p-inh significantly increased the luciferase activity of Nr4a1-WT reporter when compared to the corresponding control group $(p<0.01)$. However, the luciferase activity in Nr4a1-MUT reporter co-transfected with the miR-21-5p or miR-21-5p-inh was almost unchanged when compared to the corresponding control group (Fig. 4C). The western blot analysis disclosed that the protein level of $\mathrm{Nr} 4 \mathrm{a} 1$ was significantly decreased by miR-21-5p mimics in MPVECs $(p<0.01)$, while it was effectively increased following miR-21-5p-inh treatment $(p<0.01)$ (Fig. 4D). Then, the miR-21-5p-inh was trans- fected into H/R-induced MPVECs that were treated with Dex. The western blot analysis showed that the Dex treatment $(H / R+D e x)$ caused the decrease in the $\mathrm{Nr} 4 \mathrm{a} 1$ protein level in $\mathrm{H} / \mathrm{R}$-induced MPVECs. However, miR-21-5p-inh (H/R+Dex+miR-21-5p inh) led to a significant induction in the $\mathrm{Nr} 4 \mathrm{a} 1$ protein level when compared to the $\mathrm{NC}$ inh group $(\mathrm{H} / \mathrm{R}+\mathrm{Dex}+\mathrm{NC}$ inh) in $\mathrm{H} / \mathrm{R}$-induced MPVECs that were treated with Dex $(p<0.01)$ (Fig. 4E). These data suggested that Dex inhibited $\mathrm{Nr} 4 \mathrm{a} 1$ expression via upregulation of miR-21-5p. 
A

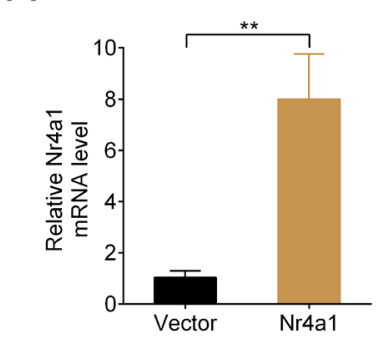

B



C



$\mathrm{D}$

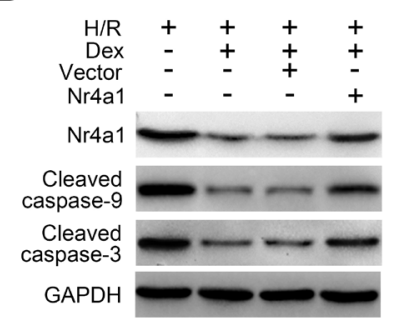

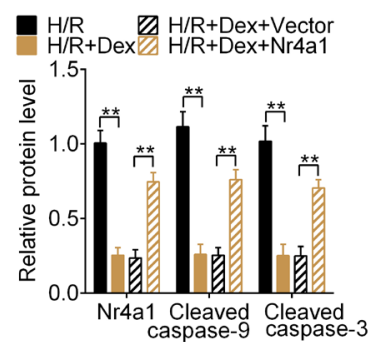

Figure 5. Involvement of Nr4a1 in the effect of DEX on H/R induced apoptosis of MPVECs.

(A) Relative expression level of Nr4a1 in MPVECs after transfection with Nr4a1 expression vector was detected by quantitative real-time reverse transcriptase polymerase chain reaction (qRT-PCR). (B) CCK-8 assay was used to detect cell viability. (C) The cells were subjected to the FACS analysis to determine the cell apoptotic ratio. (D) The protein levels of cleaved caspase-3 and cleaved caspase-9 in transfected cells were determined by western blot analysis with corresponding antibodies.The results are representative of three independent experiments. The values represent mean \pm S.D. ( $n=3$ for each group). ${ }^{* *} p<0.01,{ }^{*} p<0.05$.

\section{Dex reduced H/R-induced apoptosis of MPVECs cells by inhibiting Nr4a1 expression}

To confirm the mechanism of Dex on reducing H/Rinduced apoptosis in MPVECs, we constructed an Nr4a1 expression vector and transfected it into MPVECs. The result of qRT-PCR showed that the relative $\mathrm{Nr} 4 \mathrm{a} 1$ mRNA level was highly upregulated as compared to the control group (Fig. 5A). Then, the Nr4a1 expression vector was transfected into $H / R$ induced MPVECs that were treated with Dex. The result of CCK8 assay demonstrated that overexpression of $\mathrm{Nr} 4 \mathrm{a} 1 \mathrm{H} / \mathrm{R}+\mathrm{Dex}+$ $\mathrm{Nr} 4 \mathrm{a} 1)$ remarkably reduced the cell viability in comparison with the vector group $(\mathrm{H} / \mathrm{R}+\mathrm{Dex}+$ vector $)(p<0.01)$ (Fig. 5B). Also, the data obtained by Annexin V-FITC/ PI double staining using flow cytometry indicated that the apoptotic ratio was greatly increased by overexpression of $\mathrm{Nr} 4 \mathrm{a} 1(\mathrm{H} / \mathrm{R}+\mathrm{Dex}+\mathrm{Nr} 4 \mathrm{a} 1)$ as compared with the vector group $(\mathrm{H} / \mathrm{R}+\mathrm{Dex}+$ vector $)$ in $\mathrm{H} / \mathrm{R}$-induced MPVECs that treated with Dex $(p<0.01)$ (Fig. 5C). The results from western blot analysis indicated that the protein levels of Cleaved Caspase-3 and Cleaved Caspase- 9 were upregulated by overexpression of $\mathrm{Nr} 4 \mathrm{a} 1(\mathrm{H} /$ $\mathrm{R}+\mathrm{Dex}+\mathrm{Nr} 4 \mathrm{a} 1)$ when compared to the vector group $(\mathrm{H} / \mathrm{R}+\mathrm{Dex}+$ vector $)$ in $\mathrm{H} / \mathrm{R}$ induced MPVECs that were treated with Dex $(p<0.01)$ (Fig. 5D). These results indicated that DEX exerts anti-apoptotic effects via upregulation of miR-21-5p and subsequent downregulation of $\mathrm{Nr} 4 \mathrm{a} 1$.

\section{DISCUSSION}

PIRI is a pathological phenomenon in which tissue damage is aggravated and even irreversible damage occurs after blood flow restores in the ischemic tissues and organs (Mariscal et al., 2018). In recent years, various studies have shown that the pathogenesis of PIRI involves many pathophysiological processes and pathways, including autophagy, autoimmune oxidative stress injury and apoptosis, etc. (Zhang et al., 2003; Zhang et al., 2013; Denlinger 2015; Laubach \& Sharma 2016). In order to study the mechanism of PIRI and explore the therapeutic effect of medicines, animal models of PIRI were established ( $\mathrm{Xu}$ et al., 2018; Gu et al., 2019). In these models, the $\mathrm{W} / \mathrm{D}$ weight ratio and $\mathrm{MDA}$ were used as indirect markers of pulmonary injury. On account of that, wet pulmonary weight can indicate the pulmonary tissue damage resulting from parenchymal inflammation or immune system disorders, such as alveolar hemorrhage, congestion and pulmonary edema (Thomsen et al., 2008). As an end product of lipid peroxidation, MDA was reported to be an important precursor of oxidative injury (Aydin et al., 2009). Meanwhile, the histopathological analysis of pulmonary tissues from a mice model was also performed in respect to the rates of vascular congestion, inflammation, and thrombosis (Jacobson et al., 2005). Since the pulmonary tissue consists of various kinds of cells, it is difficult to precisely analyze the apoptotic effect of medicines in vivo. The $\mathrm{H} / \mathrm{R}$-induced MPVECs in vitro model was also established to study the apoptotic effect of medicines (Jiang et al., 2014; Liu et al., 2014). The study presented here is based on the PIRI mouse model and H/Rinduced apoptosis of MPVECs is used as an in vitro model. In the PIRI mouse model, the $\mathrm{W} / \mathrm{D}$ ratio of the pulmonary tissues was decreased, more pulmonary injury was observed, and the MDA level was increased as compared to the Sham group. These results suggested a successful construction of the PIRI mouse model. The following analysis showed that these investigations in the PIRI mouse model were reversed by Dex treatment.

Dex has been recently found to alleviate the ischemia and reperfusion induced injury in different organs (Zhang et al., 2015; Si et al., 2018; Wang et al., 2018; Zhang et al., 2019). In our present study, Dex was found to reduce PIRI by reducing the MDA level, decreasing $\mathrm{W} / \mathrm{D}$ ratio of the pulmonary tissues and attenu- 
ate pulmonary histoarchitecture injury. In addition, Dex also reduced the H/R-induced apoptosis of MPVECs, and downregulated protein levels of Cleaved Caspase-3 and Cleaved Caspase-9. Liang, et al., reported that Dex treatment could attenuate PIRI by activating the PI3K/ Akt signaling pathway at the transcriptional level (Liang et al., 2019). We found that Dex upregulated expression of miR-21-5p and downregulated expression of Nr4a1 in $\mathrm{H} / \mathrm{R}$-induced MPVCs.

Increasing evidences have reported that miRNAs play important roles in various biological processes, such as cell metabolism, proliferation and apoptosis (Diaz et al., 2017; Sun et al., 2019; Wu et al., 2019). Among them, miR-21-5p has been widely demonstrated to be dysregulated in pulmonary tumors (Wang et al., 2017; Zhong et al., 2019). Caruso, et al., described that miR-21 level was reduced in pulmonary injury induced by hypoxia challenge (Caruso et al., 2010). Li, et al., demonstrated that miR-21-5p could mediate mesenchymal stromal cellsecreted exosomes to alleviate ischemia/reperfusion injury in mouse ( $\mathrm{Li}$ et al., 2019). Our study indicated that miR-21-5p was downregulated in the $H / R$ induced MPVECs in a time-dependent manner. In addition, expression of miR-21-5p could be upregulated by Dex in a dose-dependent manner.

The orphan nuclear receptor 4A1 (Nr4a1), belonging to the steroid nuclear hormone receptor superfamily, has been shown to play a role in complex pathways of cell survival and apoptosis (Li et al., 2006). The study of Godoi, et al., reported that $\mathrm{Nr} 4 \mathrm{a} 1$ mediated cell apoptosis and autophagy via binding to the anti-apoptotic B cell lymphoma gene 2 family proteins (Godoi et al., 2016). Our study indicated that the expression of $\mathrm{Nr} 4 \mathrm{a} 1$ was upregulated in $\mathrm{H} / \mathrm{R}$ induced MPVECs in a time-dependent manner. And Dex could downregulate the expression of Nr4a1 in a dose-dependent manner. The research of Yan, et al., showed that miR-21 inhibited NR4A1 expression in the human endometrial stromal cells dagger (Yan et al., 2019). In the present study, bioinformatics analysis identified $\mathrm{Nr} 4 \mathrm{a} 1$ as a putative target for miR-21-5p, and this was confirmed by the luciferase assay. Also, knockdown of miR-21-5p could upregulate the expression of $\mathrm{Nr} 4 \mathrm{a} 1$, and overexpression of Nr4a1 reversed the protective effect of Dex on attenuating MPVECs injury induced by $\mathrm{H} / \mathrm{R}$. Taken together, these results indicated that Dex inhibited Nr4a1 expression by upregulation of miR-21-5p to reduce the apoptosis of MPVECs.

In conclusion, our study indicated a remarkable biological role of Dex in the I/R-induced pulmonary injury. This study demonstrated that Dex could attenuate I/Rinduced pulmonary injury by regulating $\mathrm{Nr} 4 \mathrm{a} 1$ expression via miR-21-5p, which thus increased cell viability and decreased cell apoptosis. Our study provides a theoretical evidence for clinical use of Dex in the therapy of pulmonary injury.

\section{Conflict of Interests}

The authors declare that they have no competing interests, and all authors confirm its accuracy.

\section{Availability of data and materials}

All data generated or analyzed during this study are included in this published article.

\section{Authors' contributions}

WD and ZDZ conceived and designed the experiments, HXY and MHC analyzed and interpreted the results of the experiments, XZ-JJY and GH performed the experiments.

\section{Ethics approval and consent to participate}

The animal use protocol described in the manuscript has been reviewed and approved by the Animal Ethical and Welfare Committee.

\section{Patient consent for publication}

Not Applicable

\section{REFERENCES}

Amour J, Cholley B, Ouattara A, Longrois D, Leprince P, Fellahi JL, Riou B, Hariri S, Latremouille C, Remy A, Provenchere S, Carillion A, Achouh P, Labrousse L, Tran Dinh A, Ait Hamou N, Charfeddine A, Lafourcade A, Hajage D, Bougle A, S investigators (2019) The effect of local anesthetic continuous wound infusion for the prevention of postoperative pneumonia after on-pump cardiac surgery with sternotomy: the STERNOCAT randomized clinical trial. Intensive Care Med 45: 33-43. https://doi.org/10.1007/s00134-0185497-x

Aydin S, Uzun H, Sozer V, Altug T (2009) Effects of atorvastatin therapy on protein oxidation and oxidative DNA damage in hypercholesterolemic rabbits. Pharmacol Res 59: 242-247. https://doi. org/10.1016/j.phrs.2009.01.004

Cao QF, Qu MJ, Yang WQ, Wang DP, Zhang MH, Di SB (2015) Ischemia postconditioning preventing lung ischemia-reperfusion injury. Gene 554: 120-124. https://doi.org/10.1016/j.gene.2014.10.009

Caruso P, MacLean MR, Khanin R, McClure J, Soon E, Southgate M, MacDonald RA, Greig JA, Robertson KE, Masson R, Denby L, Dempsie Y, Long L, Morrell NW, Baker AH (2010) Dynamic changes in lung microRNA profiles during the development of pulmonary hypertension due to chronic hypoxia and monocrotaline. Arterioscler Thromb Vasc Biol 30: 716-723. https://doi.org/10.1161/ ATVBAHA.109.202028

Clarke D, Gerard W, Norris T (2002) Pulmonary barotrauma-induced cerebral arterial gas embolism with spontaneous recovery: commentary on the rationale for therapeutic compression. Aviat Space Environ Med 73: 139-146

den Hengst WA, Gielis JF, Lin JY, Van Schil PE, De Windt LJ, Moens AL (2010) Lung ischemia-reperfusion injury: a molecular and clinical view on a complex pathophysiological process. Am J Physiol Heart Circ Physiol 299: H1283-H1299. https://doi.org/10.1152/ajpheart.00251.2010

Denlinger CE (2015) Toll-like receptor 4 inhibition in lung ischemiareperfusion injury: Time for a clinical trial? J Thorac Cardiovasc Surg 149: 1662-1663. https://doi.org/10.1016/j.jtcvs.2015.02.057

Diaz I, Calderon-Sanchez E, Toro RD, Avila-Medina J, de Rojas-de Pedro ES, Dominguez-Rodriguez A, Rosado JA, Hmadcha A, Ordonez A, Smani T (2017) miR-125a, miR-139 and miR-324 contribute to Urocortin protection against myocardial ischemia-reperfusion injury. Sci Rep 7: 8898. https://doi.org/10.1038/s41598-017-09198-x

Godoi PH, Wilkie-Grantham RP, Hishiki A, Sano R, Matsuzawa Y, Yanagi H, Munte CE, Chen Y, Yao Y, Marassi FM, Kalbitzer HR, Matsuzawa S, Reed JC (2016) Orphan nuclear receptor NR4A1 binds a novel protein interaction site on anti-apoptotic b cell lymphoma gene 2 family proteins. J Biol Chem 291: 14072-14084. https://doi.org/10.1074/jbc.M116.715235

Gu X, Yu N, Pang X, Zhang W, Zhang J, Zhang Y (2019) EXPRESS: Products of oxidative stress and TRPA1 expression in the brainstem of rats after lung ischemia-reperfusion injury. Pulm Circ 2045894019865169. https://doi.org/10.1177/2045894019865169

Huang R, Ma J, Niu B, Li J, Chang J, Zhang Y, Liu P, Luan X (2019) MiR-34b protects against focal cerebral ischemia-reperfusion (I/R) injury in rat by targeting keap1. I Stroke Cerebrovasc Dis 28: 1-9. https://doi.org/10.1016/j.jstrokecerebrovasdis.2018.08.023

Jacobson JR, Barnard JW, Grigoryev DN, Ma SF, Tuder RM, Garcia JG (2005) Simvastatin attenuates vascular leak and inflammation in murine inflammatory lung injury. Am J Physiol Lung Cell Mol Physiol. 288: L1026-L1032. https://doi.org/10.1152/ajplung.00354.2004

Jiang L, Li L, Shen J, Qi Z, Guo L (2014) Effect of dexmedetomidine on lung ischemiareperfusion injury. Mol Med Rep 9: 419-426. https://doi.org/10.3892/mmr.2013.1867

Jiang M, Xu X, Bi Y, Xu J, Qin C, Han M (2014) Systemic inflammation promotes lung metastasis via E-selectin upregulation in mouse 
breast cancer model. Cancer Biol Ther 15: 789-796. https://doi. org/10.4161/cbt.28552

Krishnadasan B, Naidu B, Rosengart M, Farr AL, Barnes A, Verrier ED, Mulligan MS (2002) Decreased lung ischemia-reperfusion injury in rats after preoperative administration of cyclosporine and tacrolimus. J Thorac Cardiovasc Surg 123: 756-767

Kucukebe OB, Ozzeybek D, Abdullayev R, Ustaoglu A, Tekmen I, Kume T (2017) Effect of dexmedetomidine on acute lung injury in experimental ischemia-reperfusion model. Braz $J$ Anesthesiol 67: 139-146. https://doi.org/10.1016/j.bjane.2015.08.002

Laubach VE, Sharma AK (2016) Mechanisms of lung ischemia-reperfusion injury. Curr Opin Organ Transplant 21: 246-252. https://doi. org/10.1097/MOT.0000000000000304

Li JW, Wei L, Han Z, Chen Z (2019) Mesenchymal stromal cells-derived exosomes alleviate ischemia/reperfusion injury in mouse lung by transporting anti-apoptotic miR-21-5p. Eur J Pharmacol 852: 6876. https://doi.org/10.1016/j.ejphar.2019.01.022

Li QX, Ke N, Sundaram R, Wong-Staal F (2006) NR4A1, 2, 3 - an orphan nuclear hormone receptor family involved in cell apoptosis and carcinogenesis. Histol Histopathol. 21: 533-540. https://doi. org/10.14670/HH-21.533

Li Y, Zhou JS, Liu B (2015) Cariporide pretreatment attenuates lung ischemia-reperfusion injury in rabbits. Sichuan Da Xue Xue Bao Yi Xue Ban 46: 394-398

Liang S, Wang Y, Liu Y (2019) Dexmedetomidine alleviates lung ischemia-reperfusion injury in rats by activating PI3K/Akt pathway. Eur Rev Med Pharmacol Sci 23: 370-377. https://doi.org/10.26355/ eurrev 20190116785

Liu J, Zhang S, Fan X, Yuan F, Dai J, Hu J (2019) Dexmedetomidine preconditioning ameliorates inflammation and blood-spinal cord barrier damage after spinal cord ischemia-reperfusion injury by down-regulation high mobility group box 1-toll-like receptor 4-nuclear factor kappaB signaling pathway. Spine (Phila Pa 1976) 44: E74-E81. https://doi.org/10.1097/BRS.0000000000002772

Liu S, Jiang M, Zhao Q, Li S, Peng Y, Zhang P, Han M (2014) Vascular endothelial growth factor plays a critical role in the formation of the pre-metastatic niche via prostaglandin E2. Oncol Rep 32: 2477-2484. https://doi.org/10.3892/or.2014.3516

Ma J, Chen Q, Li J, Zhao H, Mi E, Chen Y, Yi B, Ning J, Ma D, Lu K, Gu J (2018) Dexmedetomidine-mediated prevention of renal ischemia-reperfusion injury depends in part on cholinergic anti-inflammatory mechanisms. Anesth Analg 130: 1054-1062. https://doi. org/10.1213/ANE.0000000000003820

Mariscal A, Caldarone L, Tikkanen J, Nakajima D, Chen M, Yeung J, Cypel M, Liu M, Keshavjee S (2018) Pig lung transplant survival model. Nat Protoc 13: 1814-1828. https://doi.org/10.1038/s41596018-0019-4

Naderali E, Nikbakht F, Ofogh SN, Rasoolijazi H (2018) The role of rosemary extract in degeneration of hippocampal neurons induced by kainic acid in the rat: A behavioral and histochemical approach. $J$ Integr Neurosci 17: 19-25. https://doi.org/10.31083/JIN-170035

Oda J, Ivatury RR, Blocher CR, Malhotra AJ, Sugerman HJ (2002) Amplified cytokine response and lung injury by sequential hemorrhagic shock and abdominal compartment syndrome in a laboratory model of ischemia-reperfusion. I Trauma 52: 625-631; discussion 632. https://doi.org/10.1097/00005373-200204000-00003

Ren J, Li C, Liu Y, Liu H, Dong Z (2018) Protective effect of dexmedetomidine against myocardial ischemia-reperfusion injury in rabbits. Acta Cir Bras 33: 22-30. https://doi.org/10.1590/s0102865020180010000003

Ren W, Hou J, Yang C, Wang H, Wu S, Wu Y, Zhao X, Lu C (2019) Extracellular vesicles secreted by hypoxia pre-challenged mesenchymal stem cells promote non-small cell lung cancer cell growth and mobility as well as macrophage M2 polarization via miR-215p delivery. J Exp Clin Cancer Res 38: 62. https://doi.org/10.1186/ s13046-019-1027-0

Saito M, Chen-Yoshikawa TF, Suetsugu K, Okabe R, Takahagi A, Masuda S, Date H (2019) Pirfenidone alleviates lung ischemia-reperfusion injury in a rat model. J Thorac Cardiovasc Surg 158: 289-296. https://doi.org/10.1016/j.jtcvs.2018.08.098

Sauvant C, Schneider R, Holzinger H, Renker S, Wanner C, Gekle M (2009) Implementation of an in vitro model system for investigation of reperfusion damage after renal ischemia. Cell Physiol Biochem 24: 567-576. https://doi.org/10.1159/000257513

Si Y, Bao H, Han L, Chen L, Zeng L, Jing L, Xing Y, Geng Y (2018) Dexmedetomidine attenuation of renal ischaemia-reperfusion injury requires sirtuin 3 activation. Br J Anaesth 121: 1260-1271. https:// doi.org/10.1016/j.bja.2018.07.007

Sugimoto S, Yamamoto H, Kurosaki T, Otani S, Okazaki M, Yamane M, Toyooka S, Oto T (2019) Impact of chronic lung allograft dysfunction, especially restrictive allograft syndrome, on the survival after living-donor lobar lung transplantation compared with cadaveric lung transplantation in adults: a single-center experience. Surg Today 49: 686-693. https://doi.org/10.1007/s00595-019-01782-0

Sun XL, Zhang YL, Xi SM, Ma LJ, Li SP (2019) MiR-330-3p suppresses phosphoglycerate mutase family member 5-inducted mitophagy to alleviate hepatic ischemia-reperfusion injury. I Cell Biochem. 120: 4255-4267. https://doi.org/10.1002/jcb.27711

Thomsen RW, Riis A, Kornum JB, Christensen S, Johnsen SP, Sorensen HT (2008) Preadmission use of statins and outcomes after hospitalization with pneumonia: population-based cohort study of 29,900 patients. Arch Intern Med 168: 2081-2087. https://doi. org/10.1001/archinte.168.19.2081

van der Kaaij NP, Kluin J, Haitsma JJ, den Bakker MA, Lambrecht BN, Lachmann B, de Bruin RW, Bogers AJ (2009) Surfactant pretreatment decreases long-term damage after ischemia-reperfusion injury of the lung. Eur J Cardiothorac Surg 35: 304-312; discussion 312. https://doi.org/10.1016/j.ejcts.2008.09.050

Vora KS, Baranda U, Shah VR, Modi M, Parikh GP, Butala BP (2015) The effects of dexmedetomidine on attenuation of hemodynamic changes and there effects as adjuvant in anesthesia during laparoscopic surgeries. Saudi J Anaesth 9: 386-392. https://doi. org/10.4103/1658-354X.159461

Wagner EM, Petrache I, Schofield B, Mitzner W (2006) Pulmonary ischemia induces lung remodeling and angiogenesis. $J$ Appl Physiol (1985) 100: 587-593. https://doi.org/10.1152/japplphysiol.00029.2005

Wang P, Chen D, Ma H, Li Y (2017) LncRNA MEG3 enhances cisplatin sensitivity in non-small cell lung cancer by regulating miR21-5p/SOX7 axis. Onco Targets Ther 10: 5137-5149. https://doi. org/10.2147/OT'T.S146423

Wang X, Zhao B, Li X (2015) Dexmedetomidine attenuates isofluraneinduced cognitive impairment through antioxidant, anti-inflammatory and anti-apoptosis in aging rat. Int J Clin Exp Med 8: 1728117288

Wang Y, Wang C, Zhang D, Wang H, Bo L, Deng X (2018) Dexmedetomidine protects against traumatic brain injury-induced acute lung injury in mice. Med Sci Monit 24: 4961-4967. https://doi. org/10.12659/MSM.908133

Wang YQ, Tang YF, Yang MK, Huang XZ (2018) Dexmedetomidine alleviates cerebral ischemia-reperfusion injury in rats via inhibition of hypoxia-inducible factor-1alpha. J Cell Biochem. https://doi. org/10.1002/jcb. 28058

Wu XQ, Tian XY, Wang ZW, Wu X, Wang JP, Yan TZ (2019) miR191 secreted by platelet-derived microvesicles induced apoptosis of renal tubular epithelial cells and participated in renal ischemia-reperfusion injury via inhibiting CBS. Cell Cycle 18: 119-129. https://doi. org/10.1080/15384101.2018.1542900

Xiao Y, Zhang S, Li Q, Liu Z, Mai W, Chen W, Lei J, Hu H (2019) miR-219a-5p Ameliorates hepatic ischemia/reperfusion injury via impairing TP53BP2. Dig Dis Sci 64: 2177-2186. https://doi. org/10.1007/s10620-019-05535-4

Xu KY, Wu CY, Tong S, Xiong P, Wang SH (2018) The selective Nlrp3 inflammasome inhibitor Mcc950 attenuates lung ischemiareperfusion injury. Biochem Biophys Res Commun 503: 3031-3037. https://doi.org/10.1016/j.bbrc.2018.08.089

Xue BB, Chen BH, Tang YN, Weng CW, Lin LN (2019) Dexmedetomidine protects against lung injury induced by limb ischemia-reperfusion via the TLR4/MyD88/NF-kappaB pathway. Kaobsiung J Med Sci https://doi.org/10.1002/kjm2.12115

Yan Q, Yan G, Zhang C, Wang Z, Huang C, Wang J, Zhou J, Liu Y, Ding L, Zhang Q, Zhen X, Jiang Y, Sun H (2019) miR-21 reverses impaired decidualization through modulation of KLF12 and NR4A1 expression in human endometrial stromal cellsdagger. Biol Reprod 100: 1395-1405. https://doi.org/10.1093/biolre/ioz026

Yao X, Yao R, Yi J, Huang F (2019) Upregulation of miR-496 decreases cerebral ischemia/reperfusion injury by negatively regulating BCL2L14. Neurosi Lett 696: 197-205. https://doi.org/10.1016/j. neulet.2018.12.039

Yu J, Yang W, Wang W, Wang Z, Pu Y, Chen H, Wang F, Qian J (2019) Involvement of miR-665 in protection effect of dexmedetomidine against Oxidative Stress Injury in myocardial cells via CB2 and CK1. Biomed Pharmacother 115: 108894. https://doi. org/10.1016/j.biopha.2019.108894

Yuan F, Fu H, Sun K, Wu S, Dong T (2017) Effect of dexmedetomidine on cerebral ischemia-reperfusion rats by activating mitochondrial ATP-sensitive potassium channel. Metab Brain Dis 32: 539-546. https://doi.org/10.1007/s11011-016-9945-4

Zhang J, Jiang H, Liu DH, Wang GN (2019) Effects of dexmedetomidine on myocardial ischemia-reperfusion injury through PI3K-AktmTOR signaling pathway. Eur Rev Med Pharmacol Sci 23: 6736-6743. https://doi.org/10.26355/eurrev_201908_18565

Zhang J, Wang JS, Zheng ZK, Tang J, Fan K, Guo H, Wang JJ (2013) Participation of autophagy in lung ischemia-reperfusion injury in vivo. J Surg Res 182: e79-e87. https://doi.org/10.1016/j.jss.2012.11.014

Zhang LX, Ding F, Wang CQ, Bing Q, Zhao Z, Wang J, Zhang L (2019) MiR-181a affects myocardial ischemia-reperfusion injury in rats via regulating akt signaling pathway. Eur Rev Med Pharmacol Sci 23: 6292-6298. https://doi.org/10.26355/eurrev_201907_18451

Zhang T, Zhang F, Zhou YA, Wang YJ, Li XF, Chen DF (2003) Effect of ischemic preconditioning on cytokines during lung ischemiareperfusion injury. Zhonghua Wai Ke Za Zhi 41: 545-547 
Zhang W, Zhang J (2017) Dexmedetomidine preconditioning protects against lung injury induced by ischemia-reperfusion through inhibition of autophagy. Exp Ther Med 14: 973-980. https://doi. org/10.3892/etm.2017.4623

Zhang W, Zhang JQ, Meng FM, Xue FS (2016) Dexmedetomidine protects against lung ischemia-reperfusion injury by the PI3K/Akt/ HIF-1alpha signaling pathway. I Anesth 30: 826-833. https://doi. org /10.1007/s00540-016-2214-1

Zhang XK, Zhou XP, Zhang Q, Zhu F (2015) The preventive effects of dexmedetomidine against intestinal ischemia-reperfusion injury in Wistar rats. Iran J Basic Med Sci 18: 604-609

Zhao L, Hua C, Li Y, Sun Q, Wu W (2015) miR-525-5p inhibits ADAMTS13 and is correlated with Ischemia/reperfusion injury-induced neuronal cell death. Int J Clin Exp Med 8: 18115-18122
Zhong J, Ren X, Chen Z, Zhang H, Zhou L, Yuan J, Li P, Chen X, Liu W, Wu D, Yang X, Liu J (2019) miR-21-5p promotes lung adenocarcinoma progression partially through targeting SET/TAF-Ialpha. Life Sci 231: 116539. https://doi.org/10.1016/j.lfs.2019.06.014

Zhou L, Yao X, Chen Y (2012) Dexamethasone pretreatment attenuates lung and kidney injury in cholestatic rats induced by hepatic ischemia/reperfusion. Inflammation 35: 289-296. https://doi. org/10.1007/s10753-011-9318-4

Zhu B, Yang JR, Jia Y, Zhang P, Shen L, Li XL, Li J, Wang B (2017) Overexpression of NR4A1 is associated with tumor recurrence and poor survival in non-small-cell lung carcinoma. Oncotarget 8: 113977 113986. https://doi.org/10.18632/oncotarget.23048 